\title{
CNN Based ToF Image Processing
}

\author{
Marian-Leontin Pop ${ }^{\ddagger}$, Szilard Molnar ${ }^{\ddagger}$, Alexandru Pop ${ }^{\ddagger}$, Benjamin Kelenyi ${ }^{\ddagger}$, Levente Tamas ${ }^{\ddagger *}$, Andrei Cozma ${ }^{\S}$ \\ https: / / www youtube. com/watch?v=kANXhHwFrCo
}

\begin{abstract}
In this paper a Time of Flight (ToF) camera specific data processing pipeline is presented, followed by real life applications using artificial intelligence. These applications include use cases such as gesture recognition, movement direction estimation or physical exercises monitoring. The whole pipeline for the body pose estimation is described in details, starting from generating and training phases to the pose estimation and deployment. The final deployment targets were Nvidia Xavier NX and AGX platforms receiving data from an Analog Devices ToF camera.
\end{abstract}

Index Terms - transfer learning, ToF, python

\section{Introduction}

In recent years the evolution of deep neural networks has affected the way in which Time of Flight (ToF) images are processed. Images from ToF cameras are usually obtained as synchronized depth and infrared (IR) image pairs. The customization of the existing deep nets to the IR and depth images allows us to reuse the existing models and techniques from this emerging domain. The applications targeted are ranging from person detection, counting, activity analysis to volumetric measurements, mapping and navigation with mobile agents. In the following parts the introduction to the specific ToF imaging, custom data processing and CNN based solutions are presented [TC21]. Although for the 2D data a bunch of CNN based solutions exists, for the 3D data [GZWY20] only some base architectures were widespread such as Pointnet [QSMG17], while for the calibration between different sensing modalities can be done in an efficient way according to [FTK19].

\section{ToF specific imaging}

The 2D image processing part is a customized IR image module based on transfer learning for bounding box estimation, skeleton extraction and hardware specific model translation. The latter is relevant in order to have a light-weight embedded solution running on limited floating-point precision hardware platforms such as Jetson Nvidia Family. As the existing CNN models are mainly with the focus on colour images, thus ones has to adopt transfer learning as a method to finetune the existing $\mathrm{CNN}$ models such as VGG, MobileNet for the infrared or depth images specific to ToF cameras. This solution seemed to be effective in terms of precision

\$ Technical University of Cluj-Napoca

* Corresponding author: Levente.Tamas@aut.utcluj.ro

$\S$ Analog Devices International

Copyright $(02021$ Marian-Leontin Pop et al. This is an open-access article distributed under the terms of the Creative Commons Attribution License, which permits unrestricted use, distribution, and reproduction in any medium, provided the original author and source are credited.

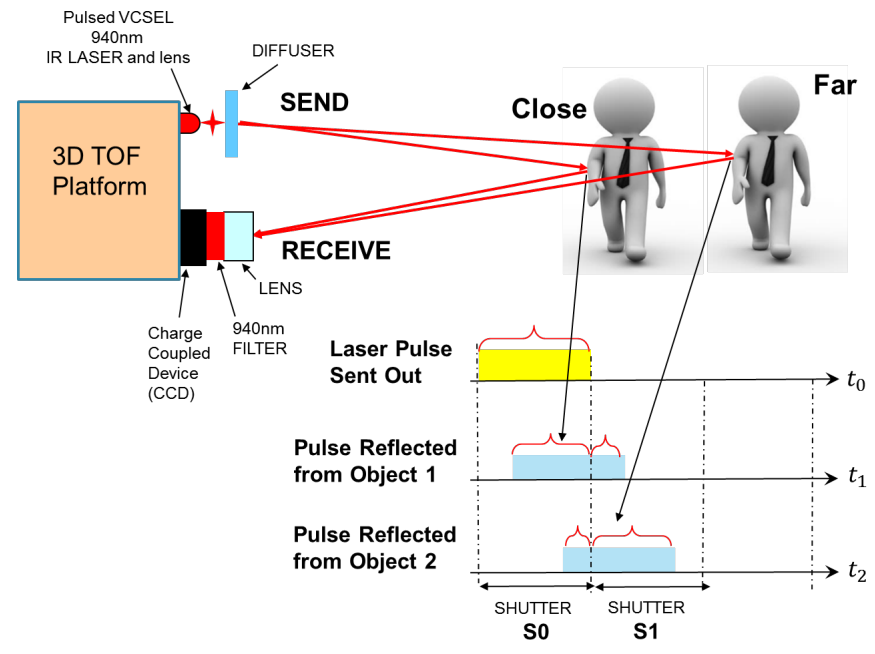

Fig. 1: Exemplification of ToF camera

and runtime on embedded devices (e.g Jetson NX or AGX). For the skeleton detection part we relied on the real-time Tensorflow optimized module for the Jetson product family, however for the generic GPU enabled devices we had to tailor our models since these are custom solutions.

\section{Custom pipeline for ToF data}

The main role of the depth image preprocessing part is the filtering and bounding box estimation for the 3D ROI. The filtering is essential for the embedded device in order to reduce the computational overload. For the filtering pipeline we considered three interconnected filters: voxel, pass-through and outlier filter as this is visible in Figure 2. All these implementations are open source library based variants. The details of the filtering were reported in [TC21].

\section{Low level ToF image pre-processing - ToFNest}

In ToFNest we are approximating surface normals from depth images, recorded with Time-of-Flight cameras. The approximation is done using a neural network. The base of our neural network is the PyTorch library, since the whole process is done using Python 3.6 as our programming language. Using PyTorch we have created a Feature Pyramid Network type model ([LDG $\left.\left.{ }^{+} 17\right]\right)$.

The main pipeline of the data was the following: first we read the depth images with OpenCV (alongside the depth information we could also use the infrared information or the rgb information from the camera as well, thus adding more information to work 


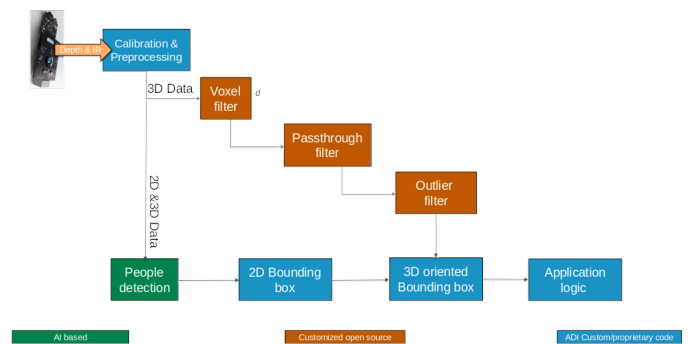

Fig. 2: Processing pipeline for ToF camera

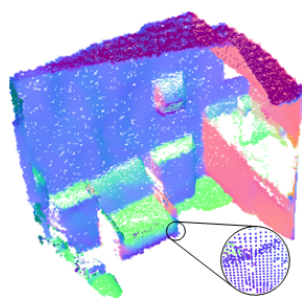

Fig. 3: Exemplification of ToF normal estimation

with), then we prepare them with numpy. From a numpy array it is easy to convert it to a torch tensor on the GPU, which then creates the predictions about the surface normals. An example of the prediction can be seen in Figure 3, where the direction of the normal vectors are decoded with RGB images.

The following code represents the loss:

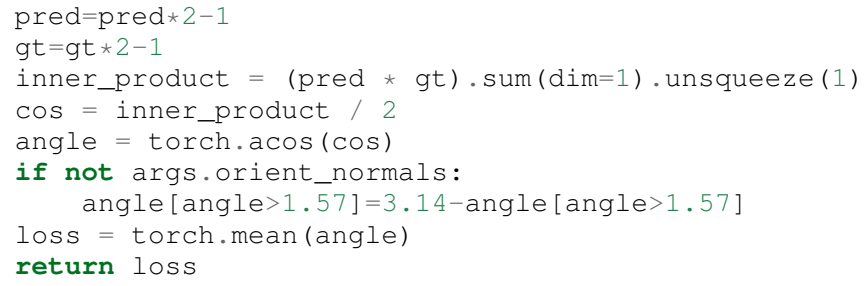

The results were accurate relative to other techniques, but the time was much less. The time being less means that at least 100 times faster. This can be due to the fact, that this method works with images, instead of point clouds as other methods do. This makes it much faster, as this was reported in [MKT21].

Our method was evaluated by verifying only the angles between the lines, not the exact directions of the vectors (this was the case in the other methods as well), but we can train that, although the results are going to get worse.

Furthermore, in order to get a real-time visualization about the predictions, we used rospy to read the images from ROS topics, and also to publish the normal estimation values to another ROS topic, that we could visualize using Rviz. This can be seen in the demo video.

\section{Low level ToF image pre-processing - ToFSmooth}

This whole pipeline and network, with some minor modifications can be also used to smoothen the depth image, thus making the point cloud smoother as well.

For the dataset we added gaussian noise of 5 and $10 \mathrm{~cm}$ to the original data, while we smoothed the original data with PointCloudDenoising ([PFVM20]) method.

Our method got pretty close to the ground truth value, in most of the cases. Although, in the case of the original (originally

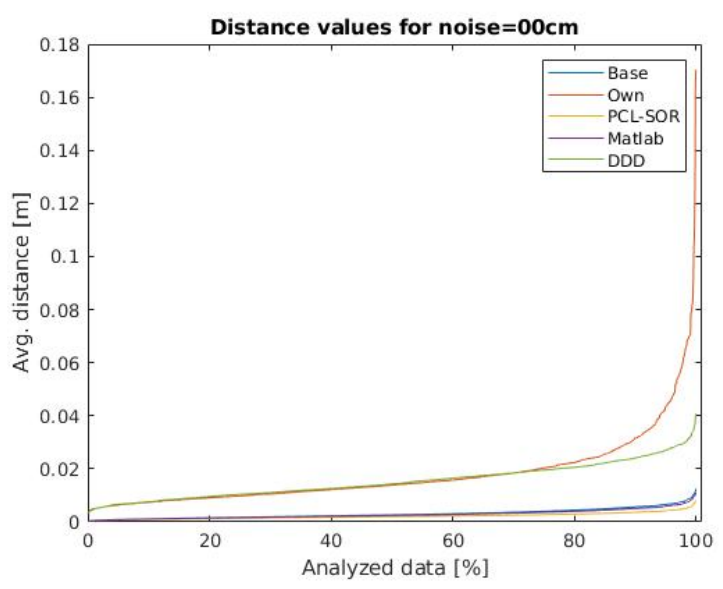

Fig. 4: The average error for the original data

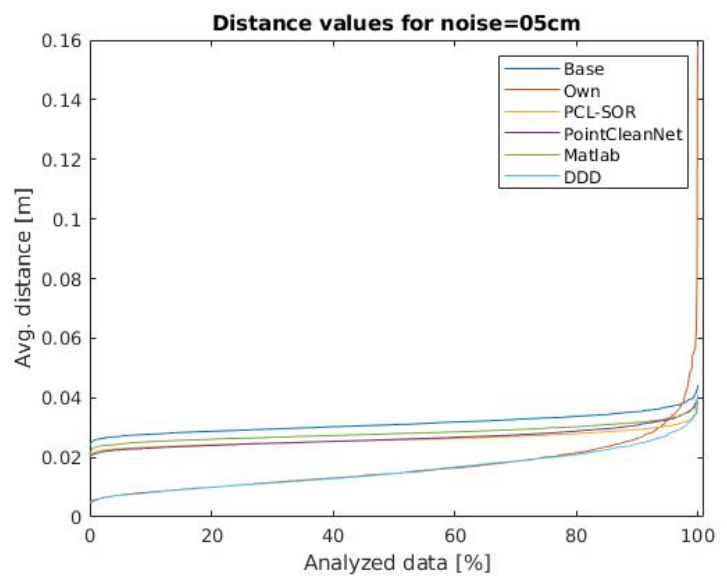

Fig. 5: The average error for data with $5 \mathrm{~cm}$ gaussian noise

fairly smooth) data resulted slightly worse results, then some other methods (for instance the PointCloud Library [RC11]), when we tested the smoothing for much more noisy data, our results barely changed, while other methods were highly compromised. A comparison between these cases can be seen in the next image 3 images:

Here we can see that our method kept very much the same

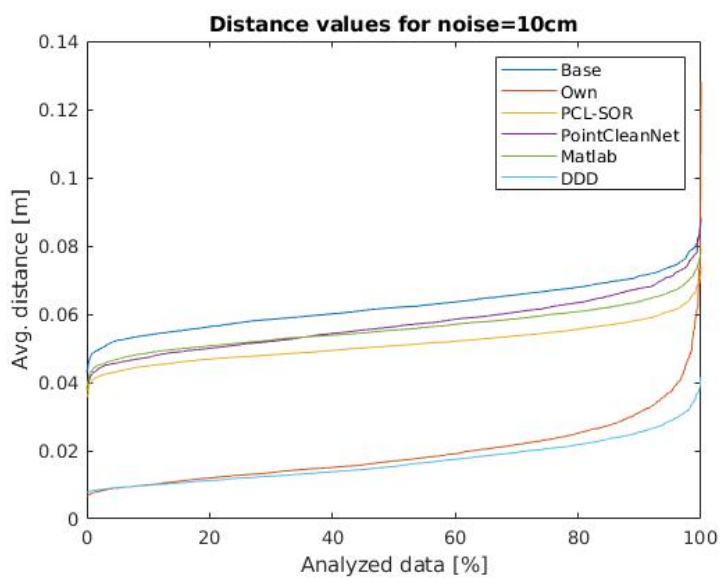

Fig. 6: The average error for data with $10 \mathrm{~cm}$ gaussian noise 


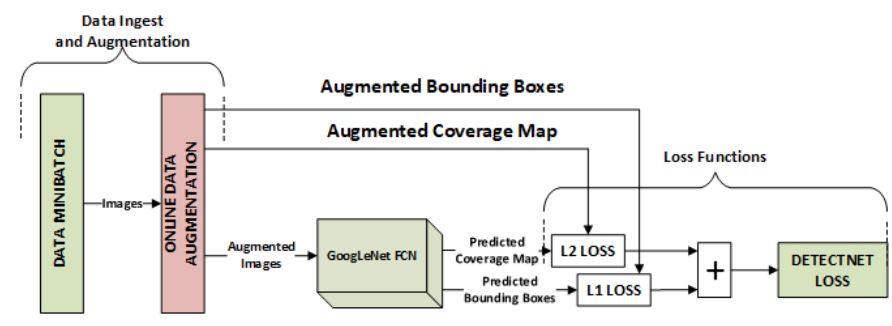

Fig. 7: DetectNet structure for training

throughout all the cases same as DeepDepthDenoising method ([SSC $\left.\left.{ }^{+} 19\right]\right)$, which is the only other method that we have found, that works with depth images as well, making it about the same as ours, but a little bit more polished. Also this method performs at the same speed as ours.

The jump in the error at the end of the scale is due to some denormalization bias that we need to fine-tune.

\section{CNN based solutions}

In this part we describe in details the person detection, action recognition and volumetric estimation applications.

\section{Person detection from IR imaging}

DetectNet is a detection algorithm based on the jetson-inference repository with people detection focus presented in $\left[\mathrm{LLW}^{+} 16\right]$ or [XLCH16]. This repository uses NVIDIA TensorRT for efficient implementation of neural networks on the Jetson platform, improving performance and energy efficiency through graphical optimizations, kernel fusion and FP16/INT8 accuracy.

Object detection requires a lot of information for training. DetectNet uses a large dataset, and each image contains multiple objects. For each object in the image, the trained model must detect both the object and the corner coordinates of the bounding box. Since the number of objects can vary in the training image set, it would be difficult to define the loss function if we choose the label format with variable length and dimensionality. This problem has been solved by introducing a 3-dimensional label format that enables DetectNet to ingest images of any size with a variable number of objects present.

In the Figure 7 you can see the architecture for the training process, which is based on 3 important steps:

- data layers ingest the training images and labels

- a fully-convolutional network (FCN) performs feature extraction and prediction of object classes and bounding boxes per grid square

- loss functions simultaneously measure the error in the two tasks of predicting the object coverage and object bounding box corners per grid square

In the final layers of DetectNet the openCV groupRectangles algorithm is used to cluster and filter the set of bounding boxes generated for grid squares with predicted coverage values greater than or equal to gridbox_cvg_threshold, which is specified in the DetectNet model definition prototxt file.

DetectNet also uses the "Python Layers" interface to calculate and output a simplified mean Average Precision (mAP) score for the final set of output bounding boxes. For each predicted bounding box and each ground truth bounding box the Intersection

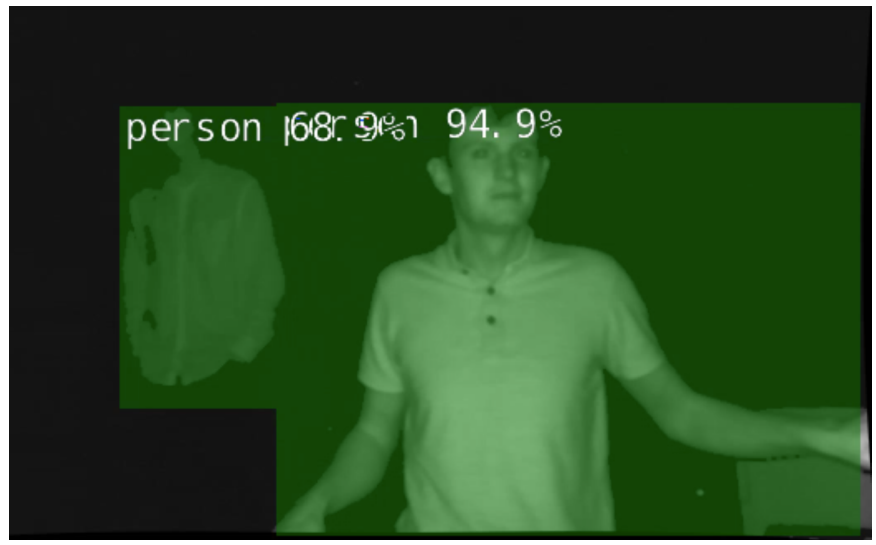

Fig. 8: Exemplification of people detection on infrared images based detection

over Union (IoU) score is computed. IoU is the ratio of the overlapping areas of two bounding boxes to the sum of their areas.

The pre-trained model accepts 3 channel images - RGB, by modifying the existing model, we have managed to detect and track people on the infrared image -1 channel. With the help of the OpenCV library and the 3.7 Python programming language version, we have developed a script that modifies the contrast of the IR image; thus, we obtained a much better result than if we had not used this approach. This result can be seen in the Figure 8 , where we can see that the people are detected on the IR image with high confidence.

To be able to run the algorithm in real-time we used the rospy client. With the help of this API, we have developed an efficient way to pass a ROS topic as input to our model. The algorithm was tested on a Jetson AGX, and the camera used was from Analog Devices (AD-96TOF1-EBZ). The result can be seen in the attached demo video.

\section{Action recognition from IR images}

This is a small tutorial for detecting the skeleton, or rather an approximation of the joints of a person, from an infrared image. In our setup we used one of the Analog Devices Time-of-Flight cameras, which provided us the infrared image, and an NVIDIA Jetson Xavier NX board, which is a compact system-on-module (SOM), very well suited for model inference.

As a baseline architecture model, we used the pretrained model from one of the NVIDIA-AI-IOT's repositories: https: //github.com/NVIDIA-AI-IOT/trt_pose . We used the TensorRT SDK in order to optimize our pretrained model for the Jetson Xavier NX platform, thus achieving a better performance in our model inference pipeline.

We also used, some of the Robot Operating System's (ROS) tools for retrieving the camera infrared images and by using the rospy client library API we managed to transfer our infrared images to the network's model. While this would have been an easy step using the CvBridge library, which provides an interface between ROS and OpenCV, this time was not the case, as we had some issues with this library. Because we are working on Jetson Xavier NX board, which comes with the latest OpenCV version, and CvBridge uses at its core an older version of OpenCv, we replaced the conversion from sensor_msgs/Image message type to the OpenCv image array made by $\mathrm{CvBridge}$ with a very useful numpy functionality which allowed us to make this conversion 


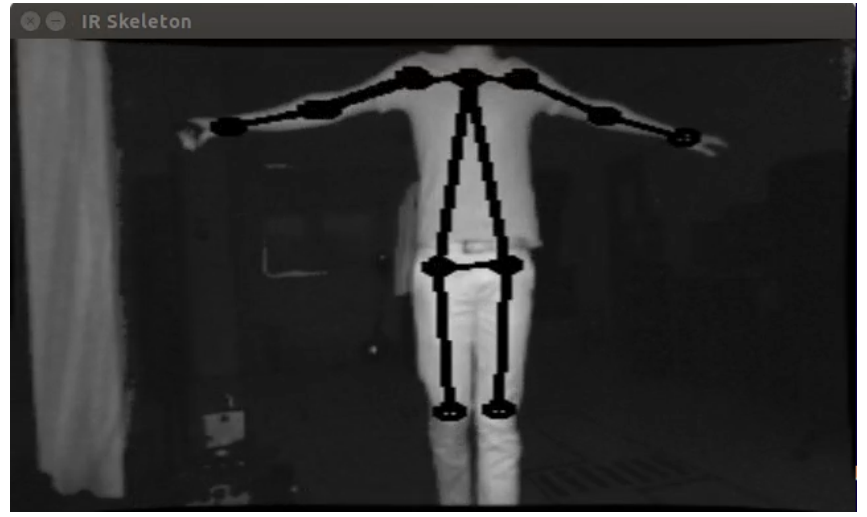

Fig. 9: Exemplification of skeleton detection on infrared images

flawlessly, while still achieving the same functionality and performance, because in fact, this was only a slight alteration of the underlying Python implementation of the CvBridge package. So, we replaced:

ir_image $=$ CvBridge ().imgmsg_to_cv2 (image_msg, -1$)$

with:

ir_image = numpy.frombuffer

image_msg.data,

dtype=numpy $\cdot$ uint 8) . reshape (

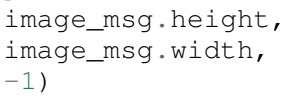

After making this conversion, we preprocessed the infrared image before feeding it to the neural network, using the OpenCv library. After this step we supply the model input with this preprocessed image, and we obtained the results which can be seen in the Figure 9.

Furthermore, as a side quest, because we tested the TensorRT SDK and we saw some good results in our model's inference, we decided to extend the infrared people detection application by integrating it with NVIDIA's Deepstream SDK. While this SDK brings further optimization to our model's inference performance and optimize the image flow along the inference pipeline by transferring the image on GPU for any kind of preprocessing required before it enters the model and even allowing us to serve multiple images, from multiple cameras, without a very drastic change in the model's inference speed. Even though these functionalities are important, we were interested by another functionality which the Deepstream SDK supports, this being the fact that is able to provide communication with a server and transmit the output of the neural network's model, which runs on the Jetson platform, to the server, for further data processing. This can be very useful in applications where we want to gather some sort of statistics or when our application has to make some decisions based on the output of our trained model, but we don't want to affect the Jetson's inference performance by overwhelming it with other processes. In the Figure 10, can be seen the result of the people detection algorithm made by using the Deepstream SDK, and below is the network's output received on our custom configured server when a person is detected:

\{

"object" :

"id" : "-1",

"speed" : 0.0 ,

"direction" : 0.0 ,

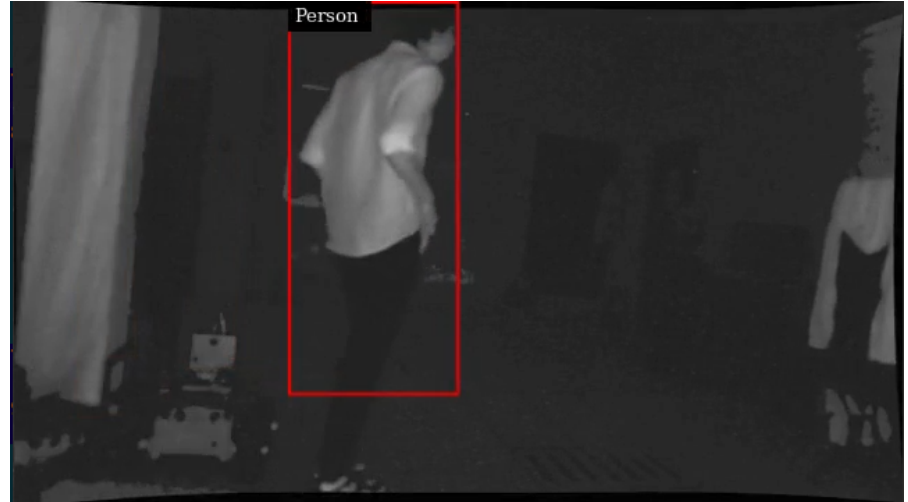

Fig. 10: People detection algorithm running with the Deepstream SDK on the Jetson Xavier NX board

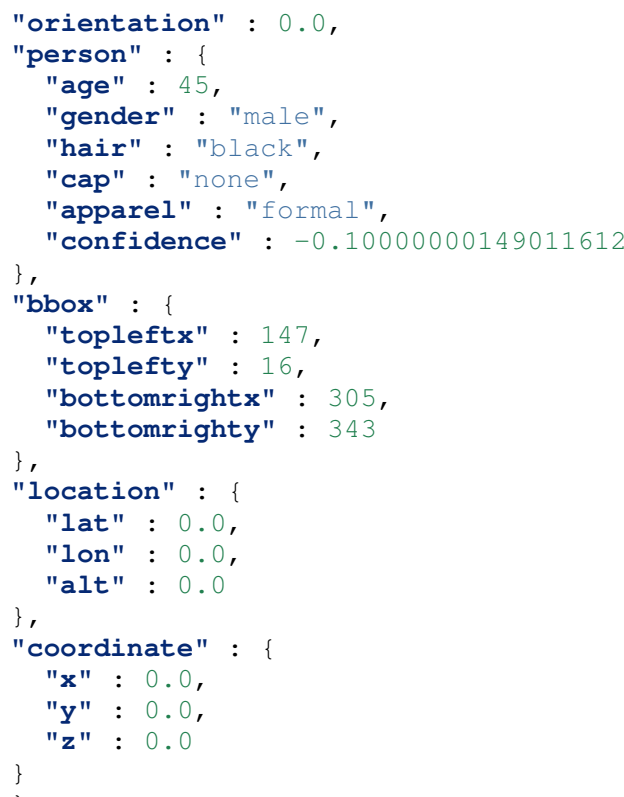

\section{Volumetric estimates for depth images}

The goal of this research is to estimate the volume of objects using only depth images recorded with Time-of-Flight cameras. As a simplifying feature, we consider only box shaped objects, with clearly definable perpendicular planes. Two methods have been determined.The first method uses RANSAC algorithm to detect planes while the other one uses the ideas from $\left[\mathrm{SSG}^{+} 20\right]$.

The first algorithm iteratively finds the largest plane using RANSAC and uses euclidean extraction to remove it from the point cloud. Once the planes are determined and checked to see if they are perpendicular, the intersection lines of the planes are determined by projecting between them. The projections approximate a line and the points with the largest component difference determine the length of the line. This way iteratively the 3 intersecting line lengths can be determined once the planes are determined and checked for orthogonality.

An important observation is that it can compute the volume using 2 planes instead of 3 . This is due to the fact that if 2 planes are orthogonal, the common line between them will be determined by 2 points that are also corner points for the object. By selecting a corner point and the two perpendicular planes, a third plane can be determined that is perpendicular to the other two and it contains 


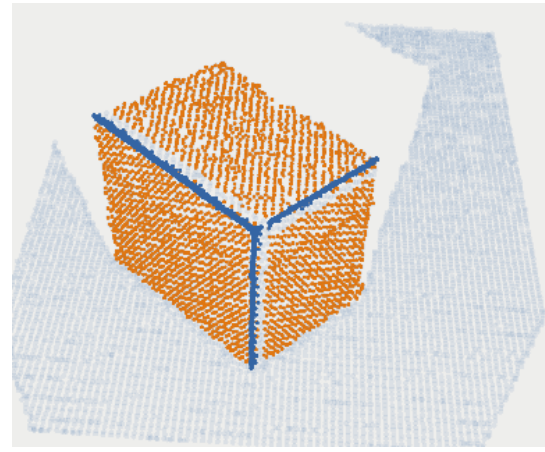

Fig. 11: Planar detection

the chosen point. Once the virtual third plane has been computed, the algorithm resumes as in the case with 3 determined planes.

An advantage of this method is that it uses readily available and studied functions for processing pointclouds. For a simple case of a box and floor plane, the algorithm accuracy depends on the level of noise the pointcloud has. The following code snippets ilustrate the functionality of the Planar Segmenting Volume computation method using 2 planes.

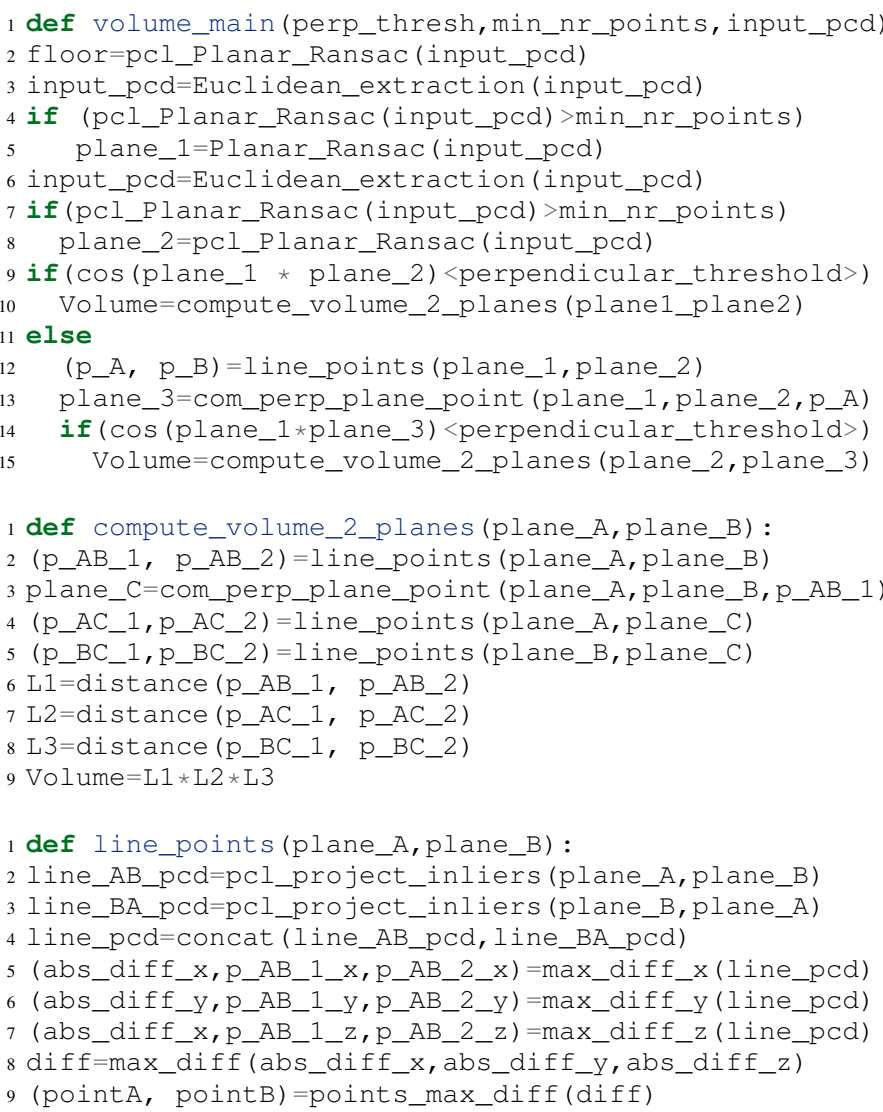

The downside of this method is that it can compute the volume only for one box. Noise and other objects in the scene can totally disrupt the volumetric estimate.

Due to these shortcomings, a new method for measuring the volume is studied, based on the work by $\left[\mathrm{SSG}^{+} 20\right]$. Their paper, details an algorithm that uses pointclouds with normals computed in each point in order to determine collections of point pairs for which their normals satisfy the orthogonality constraint. The point pair collections will approximate the orthogonal planes. By determining the points contained by each orthogonal plane,

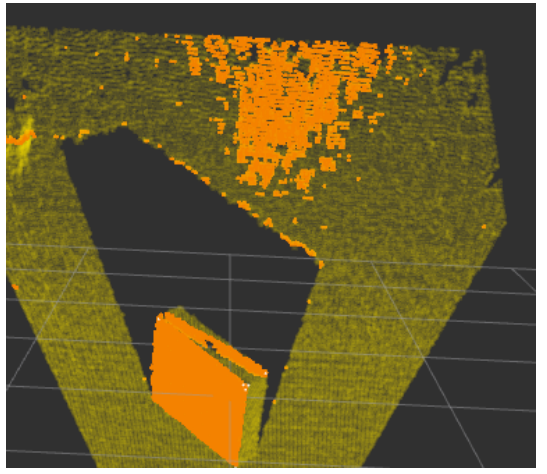

Fig. 12: Limitations of planar segmentation

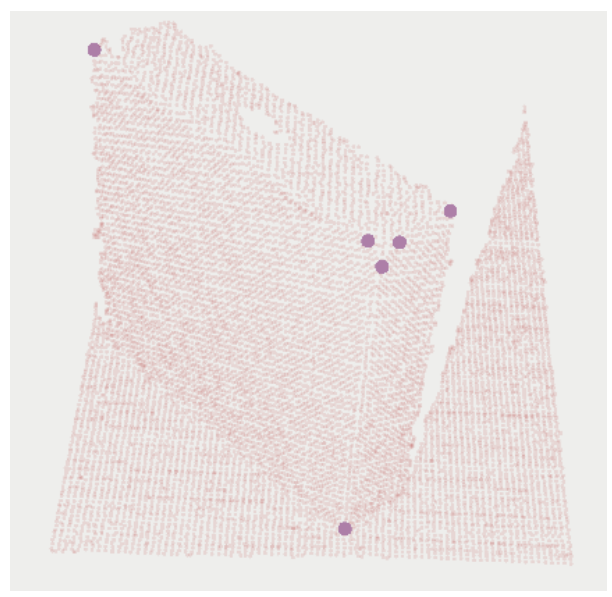

Fig. 13: Corner detection

projections can be made that approximate the intersecting lines of the orthogonal planes. By selecting the 3 lines that have the edge points closest to each other, volume of a box can be computed. The advantage of this method is that it allows the computation of the volume for multiple box shaped objects. The following code snippets show the usage of the Sommer's plane determination method to compute the volume.

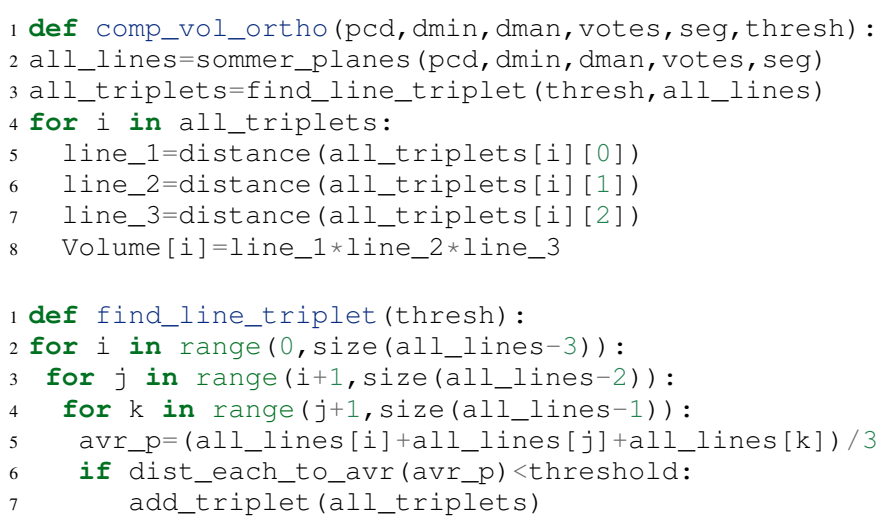

Volume estimation using enhanced planar/corner detections was done using the training from $\left[\mathrm{SSG}^{+} 20\right]$. The largest benefit of this method is that it does not rely on RANSAC and it can compute the volume for multiple objects.

This permits in further research to consider the idea of moving the camera in such a way to improve the volumetric measurement of multiple objects. This problem statement becomes equivalent to a Next Best View problem in which the view must optimize 
the accuracy or availability of a volumetric measurement. This translates to the question "In which of the available positions does the camera need to be placed next in order to improve the volumetric measurement". A starting point for such an idea would be to use the neural network architecture used in [ZZL20], but change the loss function's objective from reconstruction to volumetric accuracy. By creating a scoring function for the volumetric accuracy, candidate new positions might be evaluated and chosen based on the input pointcloud.

\section{Conclusion}

In this paper we provided some guidelines for the ToF specific image processing using Python libraries. The demos are ranging from basic pointcloud processing to people detection and enhanced volume estimation.

\section{Acknowledgement}

The authors are thankful for the support of Analog Devices Romania, for the equipment list (cameras, embedded devices, GPUs) offered as support to this work. This work was financially supported by the Romanian National Authority for Scientific Research, CNCS-UEFISCDI, project number PN-III-P2-2.1-PTE2019-0367. The authors are thankful for the generous donation from NVIDIA corporation for supporting this research.

\section{RefERENCES}

[FTK19] Robert Frohlich, Levente Tamas, and Zoltan Kato. Absolute pose estimation of central cameras using planar regions. IEEE transactions on pattern analysis and machine intelligence, 2019. doi:10.1109/TPAMI.2019.2931577.

[GZWY20] Abubakar Sulaiman Gezawa, Yan Zhang, Qicong Wang, and Lei Yunqi. A review on deep learning approaches for $3 \mathrm{~d}$ data representations in retrieval and classifications. IEEE Access, 8:5756657593, 2020. doi:10.1109/ACCESS.2020.2982196.

$\left[\mathrm{LDG}^{+} 17\right]$ T. Lin, P. Dollár, R. Girshick, K. He, B. Hariharan, and S. Belongie. Feature pyramid networks for object detection. In 2017 IEEE Conference on Computer Vision and Pattern Recognition (CVPR), pages 936-944, 2017. doi:10.1109/CVPR.2017. 106.

$\left[\mathrm{LLW}^{+} 16\right]$ Hengli Liu, Jun Luo, Peng Wu, Shaorong Xie, and Hengyu Li. People detection and tracking using rgb-d cameras for mobile robots. International Journal of Advanced Robotic Systems, 13(5):1729881416657746, 2016. doi:10.1177/ 1729881416657746.

[MKT21] Szilard Molnar, Benjamin Kelenyi, and Levente Tamas. Tofnest: Efficient normal estimation for time-of-flight depth cameras. In Proc. of IROS 2021 (under review), 2021.

[PFVM20] Francesca Pistilli, Giulia Fracastoro, Diego Valsesia, and Enrico Magli. Learning graph-convolutional representations for point cloud denoising, 2020. arXiv:2007.02578.

[QSMG17] Charles R. Qi, Hao Su, Kaichun Mo, and Leonidas J. Guibas. Pointnet: Deep learning on point sets for $3 \mathrm{~d}$ classification and segmentation, 2017. arXiv:1612.00593, doi:10.1109/ cvpr.2017.16.

[RC11] Radu Bogdan Rusu and Steve Cousins. 3D is here: Point Cloud Library (PCL). In IEEE International Conference on Robotics and Automation (ICRA), Shanghai, China, 05 2011. doi:10. 1109 /ICRA. 2011.5980567.

$\left[\mathrm{SSC}^{+} 19\right]$ Vladimiros Sterzentsenko, Leonidas Saroglou, Anargyros Chatzitofis, Spyridon Thermos, Nikolaos Zioulis, Alexandros Doumanoglou, Dimitrios Zarpalas, and Petros Daras. Selfsupervised deep depth denoising. In ICCV, 2019. doi: 10.1109 / ICCV.2019.00133.

$\left[\mathrm{SSG}^{+} 20\right]$ Christiane Sommer, Yumin Sun, Leonidas Guibas, Daniel Cremers, and Tolga Birdal. From planes to corners: Multi-purpose primitive detection in unorganized $3 \mathrm{~d}$ point clouds. IEEE Robotics and Automation Letters (RA-L), 5(2):1764-1771, 2020. doi:10.1109/LRA.2020.2969936.
[TC21]

Levente Tamas and Andrei Cozma. Embedded real-time people detection and tracking with time-of-flight camera. In Proc. of SPIE Vol, volume 11736, pages 117360B-1, 2021. doi:10. $1117 / 12.2586057$.

[XLCH16] Hongyang Xue, Yao Liu, Deng Cai, and Xiaofei He. Tracking people in rgbd videos using deep learning and motion clues. Neurocomputing, 204:70-76, 2016. Big Learning in Social Media Analytics. doi:10.1016/j.neucom.2015.06.112.

[ZZL20] Rui Zeng, Wang Zhao, and Yong-Jin Liu. Pc-nbv: A point cloud based deep network for efficient next best view planning. In 2020 IEEE/RSJ International Conference on Intelligent Robots and Systems (IROS), pages 7050-7057. IEEE, 2020. doi: 10 . 1109 /iros 45743.2020 .9340916$. 